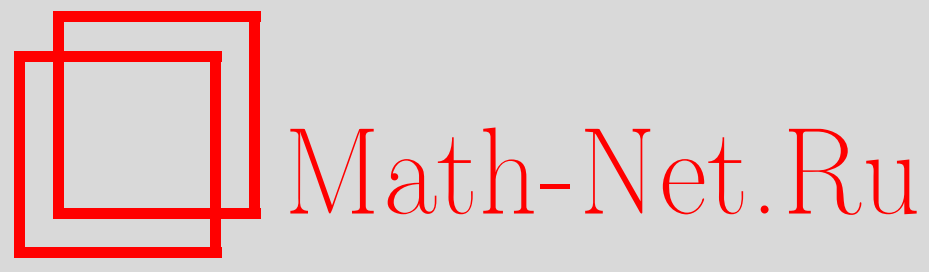

А. В. Князев, О дихотомических графах с обхватом, на единицу меньшим максимального, Дискрет. матем., 2002, том 14, выпуск 2, 119-133

DOI: https://doi.org/10.4213/dm246

Использование Общероссийского математического портала Math-Net.Ru подразумевает, что вы прочитали и согласны с пользовательским соглашением http://www.mathnet.ru/rus/agreement

Параметры загрузки:

IP : 34.227 .88 .159

26 апреля 2023 г., 11:42:06

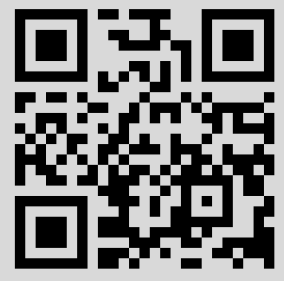


УдК 519.15

\title{
О дихотомических графах с обхватом, на единицу меньшим максимального
}

\author{
() 2002 г. А. В. Князев
}

Дихотомическим называется орграф, для полустепеней исхода $d_{0}(j)$ и захода $d_{1}(j)$ любой вершины $j \in V$ которого выполнено равенство $d_{0}(j)=d_{1}(j)=2$. Граф $Г$ называется примитивным, если для любой пары его вершин $i$ и $j$ в $\Gamma$ существует путь из $i$ и $j$ длины $m>0$. Наименьшее такое $m$ обозначается $\gamma(\Gamma)$ и называется экспонентом $\Gamma$. Обозначим через $G(n, 2, p)$ класс сильно связных дихотомических графов с $n$ вершинами и с обхватом (длиной кратчайшего контура) $p$, а через $P(n, 2, p)$ класс примитивных $n$-вершинных дихотомических графов с обхватом $p$. Обхват $n$-вершинного дихотомического графа не превосходит $] n / 2[$, где ] $x$ [ - наименьшее целое число, не меньшее $x$. Ранее автором было доказано, что любой примитивный дихотомический $n$-вершинный граф с максимально возможным обхватом ] $n / 2[$ имеет экспонент, равный в точности $n-1$.

В настоящей работе -доказано, что при нечетном $n \geqslant 13$

$$
G(n, 2,(n-1) / 2)=P(n, 2,(n-1) / 2),
$$

причем любой граф из $G(n, 2,(n-1) / 2)$ имеет контур длины $(n+1) / 2$; для любого графа $\Gamma$ из $G(n, 2,(n-1) / 2)$ справедливо неравенство

$$
\gamma(\Gamma) \leqslant \frac{(n-1)^{2}}{4}+5
$$

Напомним некоторые определения и введем обозначения. Всюду в дальнейшем под графом понимается сильно связный орграф с множеством вершин $V=\{1,2, \ldots, n\}$ без параллельных дуг.

Определение 1. Дихотомическим называется орграф, для полустепеней исхода $d_{0}(j)$ и захода $d_{1}(j)$ любой вершины $j \in V$ которого выполнено равенство $d_{0}(j)=d_{1}(j)=2$.

Определение 2. Граф Г называется примитивным, если для любой пары его вершин $i$ и $j$ в $\Gamma$ существует путь из $i$ в $j$ длины $m>0$. Наименьшее такое $m$ обозначается $\gamma(\Gamma)$ и называется экспонентом $\Gamma$.

Обозначим через $G(n, 2, p)$ класс сильно связных дихотомических графов с обхватом (длиной кратчайшего контура) $p$, а через $P(n, 2, p)$ класс примитивных дихотомических графов с обхватом $p$.

В [5] доказано, что обхват $n$-вершинного дихотомического графа не превосходит ] $n / 2$, где ] $x[$ - наименьшее целое число, не меньшее $x$. 
Обозначим через $R^{(\alpha)}$ множество всех различных подмножеств множества $V$ вершин графа $\Gamma$ мощности $\alpha$, где $1 \leqslant \alpha \leqslant n-1$. Пусть $Q \in R^{(\alpha)}$.

Расстоянием $\rho[i, Q]$ от вершины $i$ до $Q$ называется величина

$$
\rho[i, Q]=\min \left\{\rho\left(i, j_{t}\right) \mid j_{t} \in Q, t=1, \ldots, \alpha\right\},
$$

где $\rho\left(i, j_{t}\right)$ - длина кратчайшего непустого пути из $i$ в $j_{t}$ в $\Gamma$.

Определение 3. Величина

$$
D_{\alpha}(\Gamma)=\max \left\{\rho[i, Q] \mid i \in V, Q \in R^{(\alpha)}, i \notin Q\right\}
$$

называется $\alpha$-диаметром сильно связного графа $\Gamma$.

Из результатов работы [4] следует, что для любого графа $\Gamma \in G(n, 2, p)$, где $p \geqslant 3$,

$$
D_{\alpha}(\Gamma) \leqslant \frac{(n+2-\alpha)(p+1)}{2 p-1} .
$$

В [6] доказано, что для экспонента произвольного примитивного графа $\Gamma$ с обхватом (длиной наименьшего контура) $p$ справедлива достижимая верхняя оценка

$$
\gamma(\Gamma) \leqslant n+p(n-2) .
$$

В [2] доказано, что для экспонента произвольного графа $\Gamma$ из $P(n, 2, p)$ справедлива оценка

$$
\gamma(\Gamma) \leqslant \frac{n(p+1)}{2 p-1}+p(n-2)+5 .
$$

С другой стороны, в [3] доказано, что любой примитивный дихотомический граф с максимально возможным обхватом ] $n / 2$ [ имеет экспонент, равный в точности $n-1$.

В настоящей работе доказано, что при нечетном $n \geqslant 13$

$$
G(n, 2,(n-1) / 2)=P(n, 2,(n-1) / 2),
$$

причем любой граф из $G(n, 2,(n-1) / 2)$ имеет контур длины $(n+1) / 2$; для любого графа $\Gamma$ из $G(n, 2,(n-1) / 2)$ справедливо неравенство

$$
\gamma(\Gamma) \leqslant \frac{(n-1)^{2}}{4}+5 .
$$

Напомним общую идею получения верхних оценок экспонентов примитивных графов (см. [6]). Пусть $\Gamma$ - примитивный граф, и пусть $L(\Gamma)=\left\{r_{1}, \ldots, r_{m}\right\}-$ множество всех различных длин его простых контуров, где $r_{1}>\ldots>r_{m}=p$. Так как $\Gamma$ примитивен, наибольший общий делитель $\operatorname{gcd}\left(r_{1}, \ldots, r_{m}\right)$ чисел $r_{1}, \ldots, r_{m}$ равен единице.

Пусть $i$ и $j$-вершины $\Gamma$. Экспонентом $\gamma(i, j)$ пары $(i, j)$ назовем такое наименьшее натуральное $\gamma$, что для любого $q \geqslant \gamma$ существует путь из $i$ в $j$ длины $q$. Тогда

$$
\gamma(\Gamma)=\max \{\gamma(i, j) \mid i, j \in V=\{1,2, \ldots, n\}\} .
$$

Пусть $\left\{\alpha_{1}, \ldots, \alpha_{m}\right\}$ - такое множество различных натуральных чисел, что $\operatorname{gcd}\left(\alpha_{1}, \ldots, \alpha_{m}\right)=1$. Определим функцию $\varphi\left(\alpha_{1}, \ldots, \alpha_{m}\right)$ как наименьшее натуральное 
$s$ такое, что любое натуральное $q \geqslant s$ может быть представлено в виде линейной комбинации

$$
q=c_{1} \alpha_{1}+c_{2} \alpha_{2}+\ldots+c_{m} \alpha_{m},
$$

где $c_{i}, i=1, \ldots, m,-$ целые неотрицательные числа. Функция задана корректно (см. [8]), при этом в [7] доказано, что при $m=2$ справедливо равенство

$$
\varphi\left(\alpha_{1}, \alpha_{2}\right)=\left(\alpha_{1}-1\right)\left(\alpha_{2}-1\right) .
$$

Пусть $R=\left\{r_{1}, r_{2}=p\right\} \subset L(\Gamma)$ и $\operatorname{gcd}\left(r_{1}, r_{2}\right)=1, L_{i}=\left\{Q_{i}^{\left(j_{i}\right)}, j_{i}=1, \ldots, m_{i}\right\}$ - множество всех различных простых контуров длины $r_{i}, i=1,2$. Зафиксируем пару контуров $\left(Q_{1}^{\left(j_{1}\right)}, Q_{2}^{\left(j_{2}\right)}\right) \in L_{1} \times L_{2}$. Для произвольной пары вершин $i$ и $j$ в графе $\Gamma$ определим $\left(Q_{1}^{\left(j_{1}\right)}, Q_{2}^{\left(j_{2}\right)}\right)$-расстояние $\rho^{(i, j)}\left(Q_{1}^{\left(j_{1}\right)}, Q_{2}^{\left(j_{2}\right)}\right)$ как длину кратчайшего пути из $i$ в $j$, пересекающего $Q_{1}^{\left(j_{1}\right)}$ и $Q_{2}^{\left(j_{2}\right)}$ (то есть имеющего хотя бы по одной общей точке с этими контурами), если $Q_{1}^{\left(j_{1}\right)}$ и $Q_{2}^{\left(j_{2}\right)}$ не пересекаются, и пересекающего хотя бы один из пары контуров $Q_{i}^{\left(j_{i}\right)}, i=1,2$, если эти контуры пересекаются. Тогда $R$-расстояние $\rho_{R}(i, j)$ определим равенствами

$$
\rho_{R}(i, j)=\min \left\{\rho^{(i, j)}\left(Q_{1}^{\left(j_{1}\right)}, Q_{2}^{\left(j_{2}\right)}\right) \mid j_{1}=1, \ldots, m_{1}, j_{2}=1, \ldots, m_{2}\right\}
$$

Тогда для $\gamma(i, j)$ справедлива оценка

$$
\gamma(i, j) \leqslant \rho_{R}(i, j)+\varphi\left(r_{1}, r_{2}\right) .
$$

Введем обозначение

$$
\rho(R)=\max \left\{\rho_{R}(i, j) \mid i, j \in V\right\}
$$

Тогда

$$
\gamma(i, j) \leqslant \rho(R)+\varphi\left(r_{1}, r_{2}\right)
$$

Пусть $g$ - полноцикловая подстановка $(0,1, \ldots, n-1)($ a $\bar{g}-$ соответствующая ей подстановочная матрица) из симметрической группы $S_{n}$, действующей на множестве $\{0,1, \ldots, n-1\}$.

Для $g^{*} \in S_{n}$ пусть $\Delta_{j}\left(g^{*}\right)=j \ominus i$, где $g^{*}(i)=j$, а $\ominus-$ символ операции вычитания по модулю $n$, то есть $j \ominus i=j-i$, если $j \geqslant i$, и $j \ominus i=n+j-i$, если $j<i$;

$$
\begin{aligned}
\Delta_{\max }\left(g^{*}\right) & =\max \left\{\Delta_{j}\left(g^{*}\right) \mid j=0,1, \ldots, n-1\right\} \\
\Delta_{\min }\left(g^{*}\right) & =\min \left\{\Delta_{j}\left(g^{*}\right) \mid j=0,1, \ldots, n-1\right\} .
\end{aligned}
$$

Там, где будет ясно, о какой подстановке идет речь, будем писать $\Delta_{\max }, \Delta_{\min }, \Delta_{j}$.

Теорема 1. Пусть $\Gamma \in G(n, 2,(n-1) / 2)$, где $n-$ нечетное число, $n \geqslant 13$. Тогда $\Gamma$ содержит контур длины $(n+1) / 2$ и расстояние от любой вериины $\Gamma$ до контура длины $(n+1) / 2$ или $(n-1) / 2$ не превышает 2 .

Доказательство. По теореме Бирхгофа (см. [1]) для матрицы смежности $A$ графа $\Gamma$ справедливо равенство $A=\bar{g}_{1}+\bar{g}_{2}$, где $\bar{g}_{1}$ и $\bar{g}_{2}-$ подстановочные матрицы.

Возможны следующие два случая.

В первом случае хотя бы одна из подстановок $g_{1}, g_{2}$ имеет цикловую структуру $[(n-1) / 2,(n+1) / 2]$. Ясно, что в этом случае теорема 1 верна. 


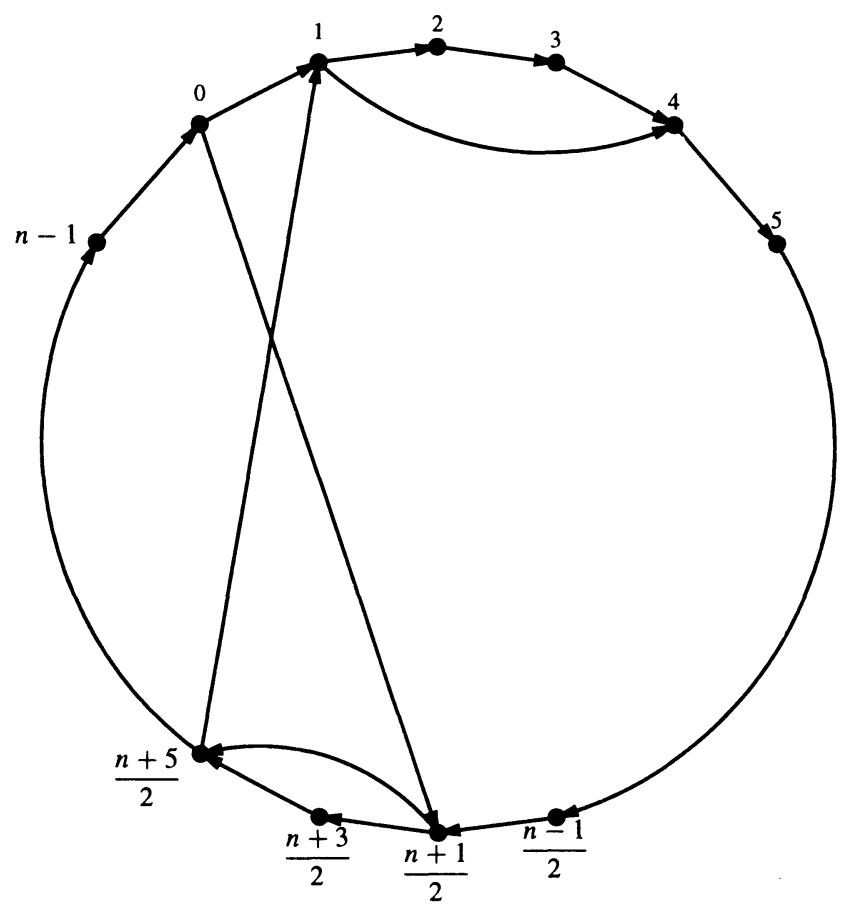

Puc. 1.

Во втором случае обе подстановки $g_{1}$ и $g_{2}$ полноцикловые. Без ограничения общности можно считать, что $g_{1}=g$ и $g_{2}=\left(0, i_{1}, i_{2}, \ldots, i_{n-1}\right)$. Так как обхват графа $\Gamma$ равен $(n-1) / 2$, справедлива оценка $\Delta_{\max }\left(g_{2}\right) \leqslant(n+1) / 2+1$ (см. рис. 1$)$.

Поскольку в $\Gamma$ нет параллельных дуг, справедлива оценка $\Delta_{\min }\left(g_{2}\right) \geqslant 2$.

Утверждения теоремы будут следовать из лемм 1-3, доказываемых ниже.

Лемма 1. Пусть в условиях теоремы $1 \Delta_{\max }\left(g_{2}\right)=(n+1) / 2$. Тогда справедливы оба утверждения теоремы 1.

Доказательство. Не ограничивая общности можно считать, что $\Delta_{\max }=\Delta_{i_{1}}$ и, значит, $g_{2}(0)=(n+1) / 2$ (см. рис. 1$)$. Тогда контур $(0,(n+1) / 2,(n+3) / 2, \ldots, n-1,0)($ см. рис. 1$)$ имеет длину $(n+1) / 2$, откуда следует справедливость первого утверждения теоремы.

Докажем второе утверждение. Ясно, что $g_{2}((n+1) / 2) \in\{1,2,(n+5) / 2\}$, иначе в $\Gamma$ существовал бы контур длины, строго меньшей $(n-1) / 2$ (или параллельные дуги).

Если $g_{2}((n+1) / 2)$ равно 1 или 2, то второе утверждение справедливо, так как контуры $(1,2, \ldots,(n+1) / 2,1)$ и $(2,3, \ldots,(n+1) / 2,2)$ имеют длины $(n+1) / 2$ и $(n-1) / 2$, соответственно. Во втором случае единственная вершина, не лежащая на контурах $(0,(n+1) / 2,(n+3) / 2, \ldots, n-1,0)$ длины $(n+1) / 2$ и $(2,3, \ldots,(n+1) / 2,2)$ длины $(n-1) / 2$ - это вершина 1 . В этом случае по пути длины 1 из вершины 1 попадаем в вершину 2 , принадлежащую контуру длины $(n-1) / 2$.

Пусть $g_{2}((n+1) / 2)=(n+5) / 2$. Тогда $g_{2}((n+5) / 2) \in\{1,2,3\}$ (см. рис. 1$)$, иначе в $\Gamma$ существовал бы контур длины, меньшей $(n-1) / 2$.

Если $g_{2}((n+5) / 2)$ равно 2 или 3 , то лемма 1 справедлива, поскольку контуры $(2,3, \ldots,(n+1) / 2,(n+5) / 2,2)$ и $(3,4, \ldots,(n+1) / 2,(n+5) / 2,3)$ имеют длины $(n+1) / 2$ 


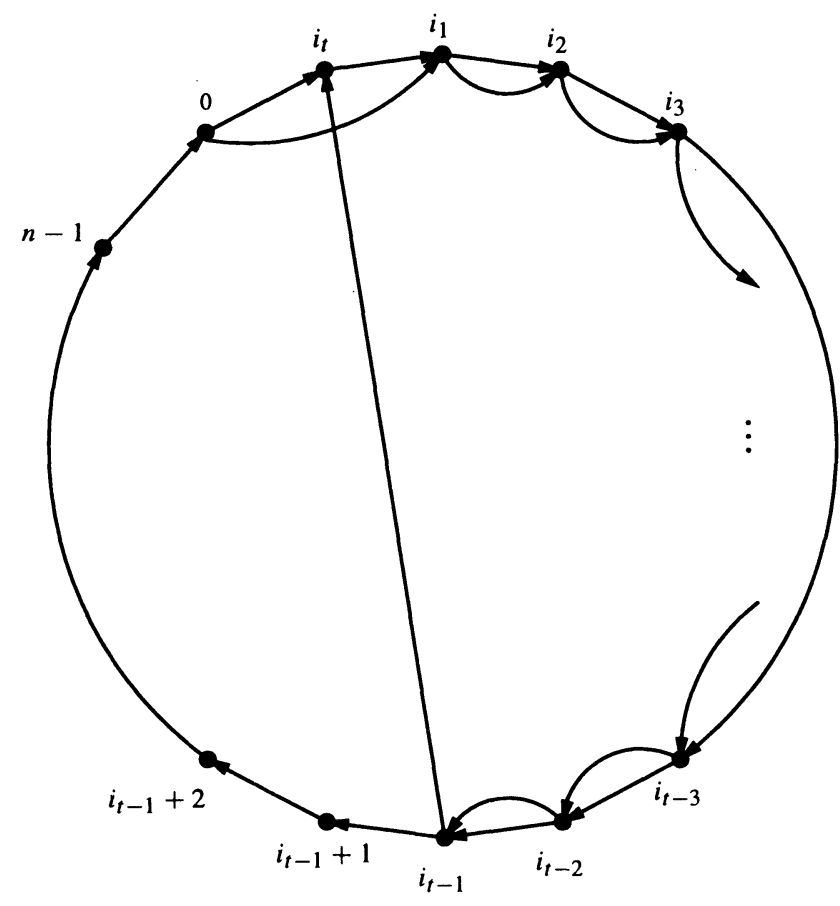

Рис. 2.

и $(n-1) / 2$ соответственно. Тогда существуют только множества вершин $\{1\}$ и $\{1,2\}$, соответственно, не лежащие на контурах соответствующих длин. Легко видеть, что максимальная длина необходимого пути (из вершины 1 в вершину 3) в рассматриваемых случаях равняется 2 .

Пусть $g_{2}((n+5) / 2)=1$. Тогда $g_{2}(1) \in\{3,4\}$. В то же время контуры

$$
(1,3,4, \ldots,(n+1) / 2,(n+5) / 2,1), \quad(1,4,5, \ldots,(n+1) / 2,(n+5) / 2,1)
$$

в соответствующих случаях имеют длины $(n+1) / 2$ и $(n-1) / 2$, соответственно. Легко видеть, что максимальная длина необходимого пути в рассматриваемых случаях (из вершины 2 в вершину 4 (см. рис. 1)) равна 2.

Лемма 1 доказана.

Лемма 2. Пусть в условиях теоремы $1 \Delta_{\max }<(n+1) / 2$. Тогда справедливы оба утверждения теоремы 1.

Доказательство. Без ограничений общности можем считать, что $\Delta_{\max }=\Delta_{i_{1}}$.

Пусть $t$ - такое наименьшее число, что

$$
g_{2}^{t}(0)=i_{t} \in\left\{0,1,2, \ldots, i_{1}\right\}
$$

(см. рис. 2). Так как $\Delta_{i_{1}}=\Delta_{\max }$, то $0<i_{1}<i_{2}<\ldots<i_{t-2}, i_{t-1}$. Из полноцикловости $g_{2}$ следует, что $i_{t} \neq 0, i_{1}$.

Рассмотрим контур $Q_{1}$ вида

$$
\left(0, i_{1}, i_{2}, \ldots, i_{t-1}, i_{t-1}+1, i_{t-1}+2, \ldots, n-1,0\right)
$$




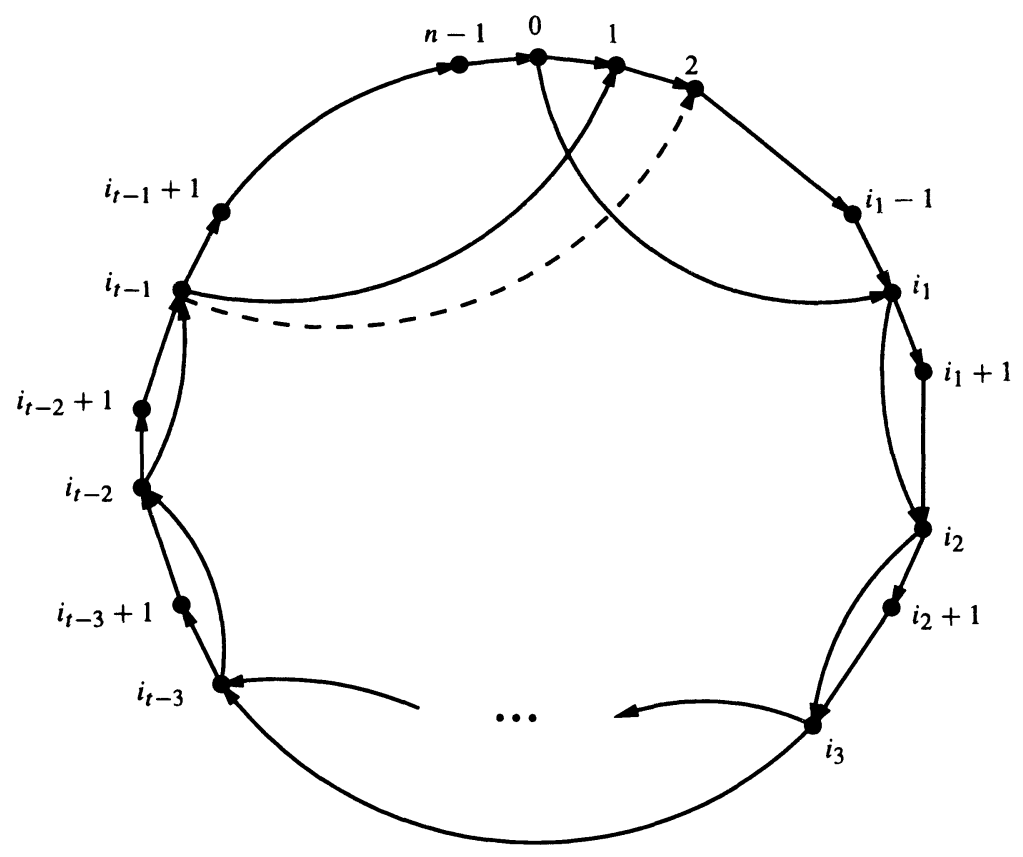

Рис. 3.

и оценим сверху его длину $l$. Ясно, что $2 \leqslant \Delta_{\min } \leqslant \Delta_{i_{j}}, j=2, \ldots, t$. Поэтому

$$
l \leqslant f\left(x, \Delta_{i_{1}}, \Delta_{i_{t}}, i_{t}\right)=1+\frac{1}{2}\left(n-\Delta_{i_{1}}-\left(\Delta_{i_{t}}-i_{t}\right)\right)-x+\Delta_{i_{t}}-i_{t},
$$

где $x$ - целое неотрицательное число.

Ясно, что $f\left(x, \Delta_{i_{1}}, \Delta_{i_{t}}, i_{t}\right)$ достигает максимума при

$$
x=0, \quad \Delta_{i_{t}}=\Delta_{i_{1}}=\Delta_{\max }, \quad i_{t}=1 .
$$

В этом случае $f\left(x, \Delta_{i_{1}}, \Delta_{i_{t}}, i_{t}\right)=f\left(\Delta_{\max }\right)=(n+1) / 2$.

Учитывая четность числа $n-2 \Delta_{\max }+1$, непосредственно проверяем, что $x=0$ тогда и только тогда, когда

$$
\Delta_{i_{j}}=2, \quad j=2, \ldots, t-1
$$

Пусть $l=(n+1) / 2$ и выполнено первое утверждение теоремы 1 . Тогда контур $Q_{2}$ вида $\left(1,2, \ldots, i_{1}-1, i_{1}, i_{2}, \ldots, i_{t-1}, 1\right)$ (см. рис. 3) имеет, как и контур $Q_{1}$, длину $(n+1) / 2$ и второе утверждение выполнено, поскольку все вершины $\Gamma$ лежат на контуре $Q_{1}$ или $Q_{2}$.

Пусть $l<(n+1) / 2$. Если $l \leqslant(n+1) / 2-2$, это противоречит условию теоремы 1 , поскольку обхват $\Gamma$ равен $(n-1) / 2$. Следовательно, $l=(n-1) / 2$.

Рассмотрим условия, при которых $f\left(x, \Delta_{i_{1}}, \Delta_{i_{t}}, i_{t}\right)=(n-1) / 2$.

Перепишем функщию $f$ в виде

$$
f\left(x, \Delta_{i_{1}}, \Delta_{i_{t}}, i_{t}\right)=1+\frac{1}{2} n-\frac{1}{2} i_{t}-\frac{1}{2}\left(\Delta_{i_{1}}-\Delta_{i_{t}}\right)-x=\frac{n-1}{2} .
$$


Тогда возможны следующие случаи:

1. $x=1, i_{t}=1, \Delta_{i_{1}}=\Delta_{i_{t}}=\Delta_{\max }$;

2.1. $x=0, i_{t}=1, \Delta_{i_{1}}=\Delta_{i_{t}}+2$;

2.2. $x=0, i_{t}=2, \Delta_{i_{1}}=\Delta_{i_{t}}+1$;

2.3. $x=0, i_{t}=3, \Delta_{i_{1}}=\Delta_{i_{t}}$.

Рассмотрим каждый из этих случаев.

В случае $1 x=1, i_{t}=1, \Delta_{i_{1}}=\Delta_{i_{t}}=\Delta_{\max }$. С учетом четности $n-2 \Delta_{\max }+1$, в этом случае непосредственно проверяется, что $x=1$ тогда и только тогда, когда

$$
\begin{aligned}
\Delta_{i_{m}} & =\Delta_{i_{q}}=3, & & 2 \leqslant m<q \leqslant t-1, \\
\Delta_{i_{j}} & =2, & & j=2,3, \ldots, m-1, m+1, \ldots, q-1, q+1, \ldots, t-1,
\end{aligned}
$$

либо

$$
\begin{aligned}
& \Delta_{i_{m}}=4, \quad 2 \leqslant m \leqslant t-1, \\
& \Delta_{i_{j}}=2, \quad j=2,3, \ldots, m-1, m+1, \ldots, t-1 \text {. }
\end{aligned}
$$

Ясно, что контуры $Q_{1}=\left(0, i_{1}, i_{2}, \ldots, i_{t-1}, i_{t-1}+1, i_{t-1}+2, \ldots, n-1,0\right)$ (см. рис. 2) и $Q_{2}=\left(1,2, \ldots, i_{1}-1, i_{1}, i_{2}, \ldots, i_{t-1}, 1\right)$ (см. рис. 3) имеют в этом случае одинаковую длину $(n-1) / 2$. Поскольку $\Delta_{1}=\Delta_{i_{1}}=\Delta_{\max }$, повторяя рассуждения, начиная с соотношения, аналогичного (4) для вершины $i_{t-1}$ (вместо вершины 0), с учетом условия, аналогичного условию (7), получаем, что $g_{2}(1)-1 \in\{3,4\}$.

Если $g_{2}(1)<i_{1}$, то в $\Gamma$ существует контур длины, меньшей, чем длина контура $Q_{2}$, что противоречит условию теоремы 1. Следовательно, $\Delta_{\max } \in\{3,4\}$.

Пусть $\Delta_{\max }=3$. Тогда, с учетом $(7), i_{1}=3, g_{2}(1)=4, g_{2}(2)=5, g_{3}(3)=6, g_{2}(4)=7$, $g_{2}(5)=8, g_{2}(6)=9$ и $g_{2}(9)=1$. При этом $\Delta_{\max }=\Delta_{\min }=3$ и $n=11$ (см. рис. 4 ). Это противоречит условию $n \geqslant 13$.

Аналогично, для $\Delta_{\max }=4$ получаем, что

$$
i_{1}=4, \quad g_{2}(1)=5, \quad g_{2}(2)=6, \quad g_{2}(3)=7, \quad g_{2}(4)=8, \quad g_{2}(8)=1 .
$$

При этом $\Delta_{\max }=\Delta_{\min }=4$ и $n=11$ (см. рис. 5). Это противоречит условию $n \geqslant 13$. Следовательно, данный случай невозможен.

В случае $2.1 x=0, i_{t}=1, \Delta_{i_{1}}=\Delta_{i_{t}}+2$. С учетом четности в этом случае $\left(n-\Delta_{i_{1}}-\left(\Delta_{i_{1}}-i_{t}\right)\right)$ получаем, что $x=0$ тогда и только тогда, когда выполнено (6). Поскольку $\Delta_{i_{1}}=\Delta_{\max }<(n+1) / 2, i_{1} \neq i_{t-1}$ (см. рис. 3). В этом случае контур $Q_{2}$ имеет длину $(n+3) / 2$.

Тогда возможны следующие случаи (a) и (b).

В случае (a) $g_{2}(1)<i_{t}$. Тогда $g_{2}(1) \in\{3,4\}$, и контуры $\left(1,3,4, \ldots, i_{1}, i_{2}, \ldots, i_{t-1}, 1\right)$ и $\left(1,4,5, \ldots, i_{1}, i_{2}, \ldots, i_{t-1}, 1\right)$ имеют длину $(n+1) / 2$ и $(n-1) / 2$, соответственно (во втором случае контур $\left(1,4,5, \ldots, i_{1}, i_{1}+1, i_{2}, i_{3}, \ldots, i_{t-1}, 1\right)$ имеет длину $\left.(n+1) / 2\right)$. В обоих случаях максимальное расстояние от вершины 2 до ближайшей вершины (3 или $4)$, лежащей на контуре длины $(n-1) / 2$ или $(n+1) / 2$, не превосходит 2 . Таким образом, лемма 2 в этом случае справедлива. 


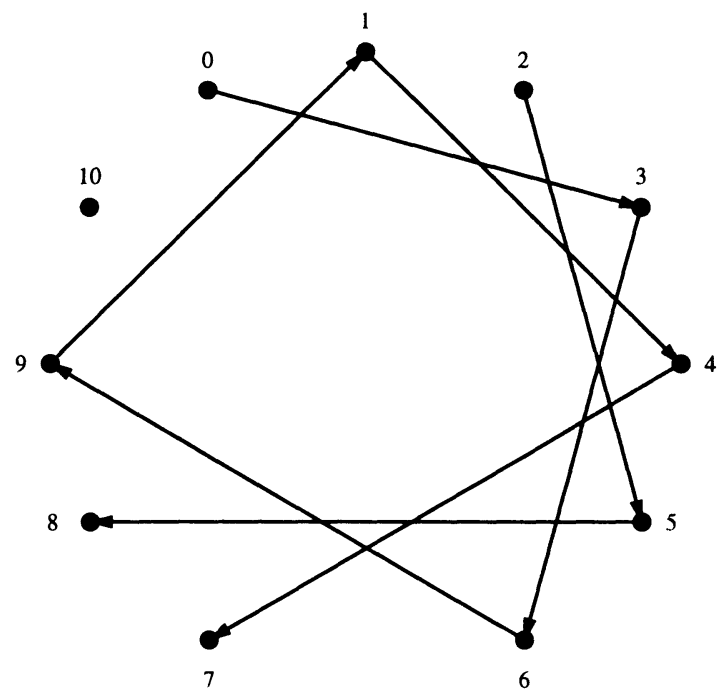

Рис. 4.

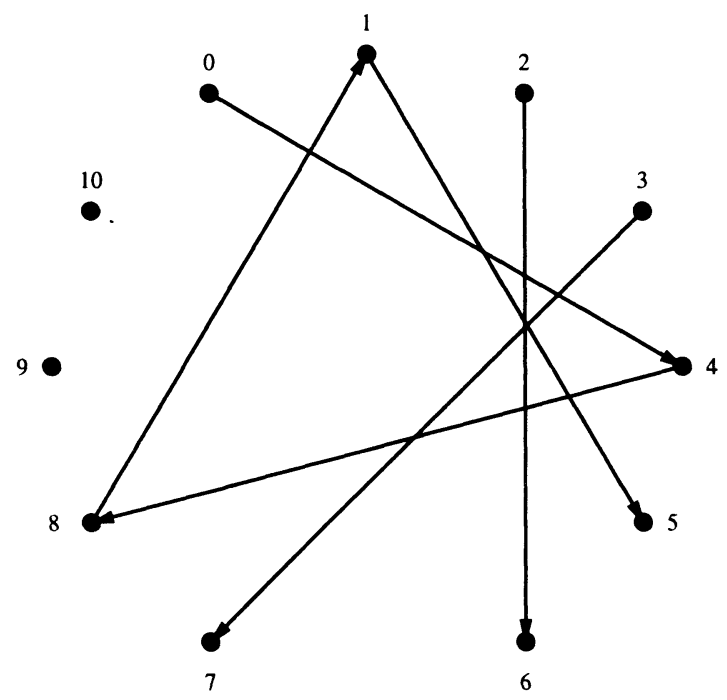

Рис. 5.

В случае (b) $g_{2}(1)>i_{1}$. Тогда $g_{2}(1)=i_{1}+1$, поскольку $\Delta_{i_{1}}=\Delta_{\max }$. Если при этом $g_{2}(2)<i_{1}$, то справедливы рассуждения, аналогичные рассуждениям пункта (а), и лемма 2 в этом случае также справедлива.

Пусть $g_{2}(2)>i_{1}$. Тогда $g_{2}(2)>i_{2}$ и $\Delta_{g_{2}(2)}>\Delta_{i_{1}}$, что противоречит исходному предположению, что $\Delta_{i_{1}}=\Delta_{\max }$.

В случае $2.2 x=0, i_{t}=2, \Delta_{i_{1}}=\Delta_{i_{t}}+1$. С учетом четности в этом случае $\left(n-\Delta_{i_{1}}-\left(\Delta_{i_{t}}-i_{t}\right)\right)$ получаем, что $x=0$ тогда и только тогда, когда выполнено (6). Поскольку $\Delta_{i_{1}}=\Delta_{\max }<(n+1) / 2, i_{1} \neq i_{t-1}$ (см. рис. 3). В этом случае контуры 


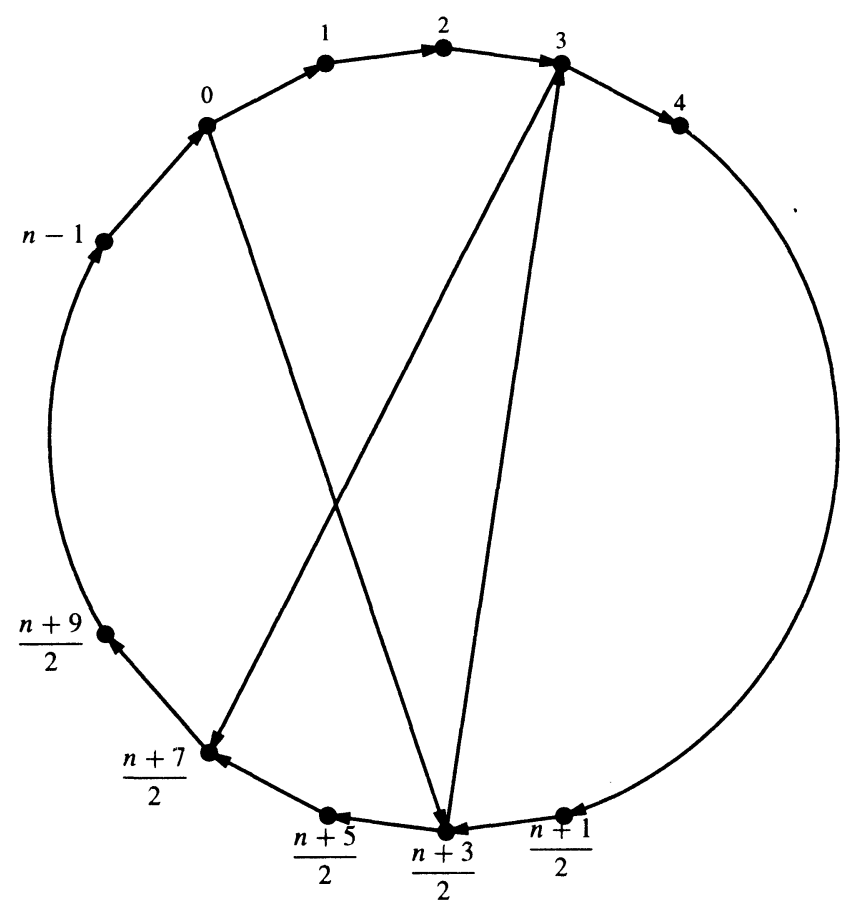

Рис. 6.

$\left(2,3, \ldots, i_{1}, i_{2}, \ldots, i_{t-1}, 2\right)$ и $\left(2,3, \ldots, i_{1}, i_{1}+1, i_{2}, \ldots, i_{t-1}, 2\right)$ имеют длину $(n-1) / 2$ и $(n+1) / 2$, и с учетом наличия контура

$$
Q_{1}=\left(0, i_{1}, i_{2}, \ldots, i_{t-1}, i_{t-1}+1, i_{P} t-1+2, \ldots, n-1,0\right)
$$

длины $(n-1) / 2$, единственная вершина, не лежащая на контуре длины $(n-1) / 2$ или $(n+1) / 2$, это вершина 1 . Следовательно, лемма 2 в этом случае справедлива.

В случае $2.3 x=0, i_{t}=3, \Delta_{i_{1}}=\Delta_{i_{t}}$ и длина контура $\left(3,4, \ldots, i_{1}, i_{2}, \ldots, i_{t-1}, 3\right)$ равна $(n-5) / 2$, что противоречит условию теоремы 1 .

Лемма 2 доказана.

Лемма 3. Пусть в условиях теоремы $1 \Delta_{\max }\left(g_{2}\right)=(n+1) / 2+1$. Тогда справедливы оба утверждения теоремы 1.

Доказательство. Без ограничений общности можем полагать, что $\Delta_{\max }=\Delta_{i_{1}}$. Тогда $g_{2}(0)=(n+1) / 2+1$ (см. рис. 6). Ясно, что $g_{2}((n+3) / 2) \in\{1,2,3\}$, в противном случае обхват Г был бы меньше $(n-1) / 2$.

Рассмотрим возможные случаи.

1. Пусть $g_{2}((n+3) / 2)=3$ (см. рис. 6$)$.

Если $g_{2}(3) \in\{0,1,2\}$, то контур $\left\{3, g_{2}(3), g_{2}(3)+1, \ldots, 3\right\}$ имеет длину, не большую 4 , что противоречит условию теоремы 1 .

Если $g_{2}(3) \in\{5,6, \ldots,(n+1) / 2\}$, то контур $\left\{3, g_{2}(3), \ldots,(n+3) / 2,3\right\}$ имеет длину, не большую $(n-3) / 2$, что противоречит условию теоремы 1. 


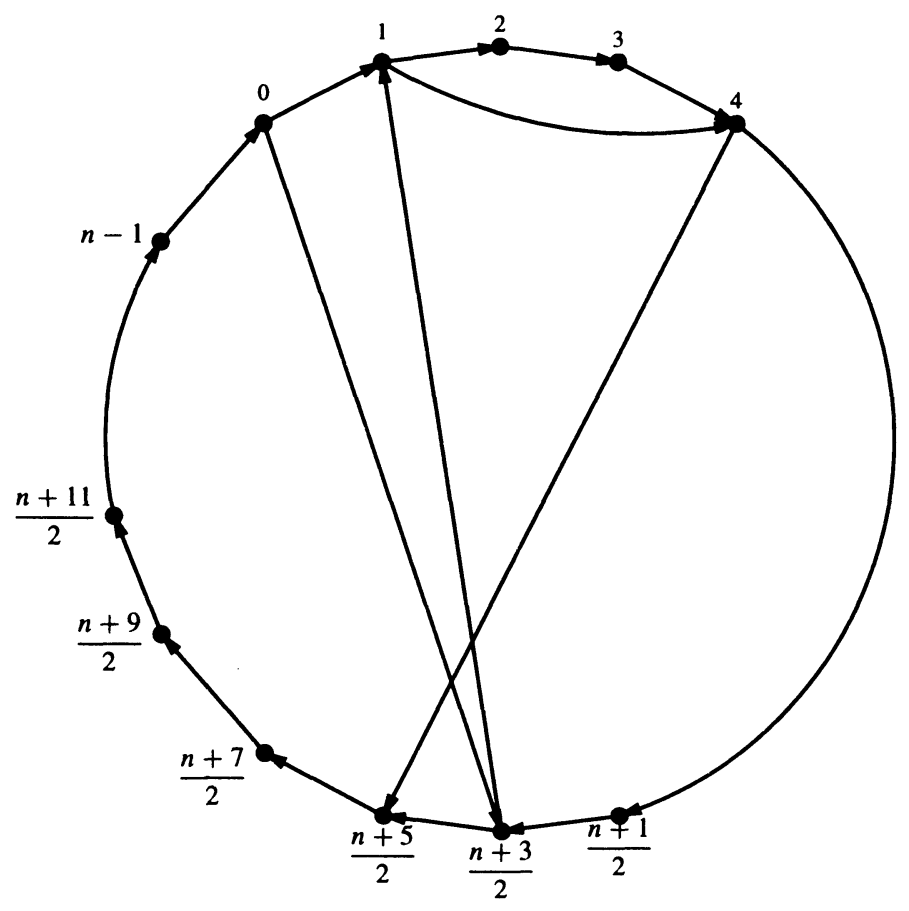

Рис. 7.

Если $g_{2}(3) \in\{(n+9) / 2,(n+11) / 2, \ldots, n-1\}$, то контур

$$
\left\{0,(n+3) / 2,3, g_{2}(3), g_{2}(3)+1, \ldots, n-1,0\right\}
$$

имеет длину, не большую $(n-3) / 2$, что противоречит условию теоремы 1 .

Если $g_{2}(3)=(n+5) / 2$, то контур $(0,(n+3) / 2,3,(n+5) / 2,(n+7) / 2, \ldots, n-1,0)$ имеет длину $(n+1) / 2$, и поскольку контур $(3,4, \ldots,(n+3) / 2,3)$ имеет длину $(n-1) / 2$, лемма 3 в этом случае справедлива.

Если $g_{2}(3)=(n+7) / 2$ (см. рис. 6$)$, то контур

$$
(3,(n+7) / 2,(n+9) / 2, \ldots, n-1,0,1,2,3)
$$

имеет длину $(n+1) / 2$, и, как и в предыдущем случае, лемма 3 в этом случае справедлива.

2 . Пусть $g_{2}((n+3) / 2)=2$. Тогда контур $(2,3, \ldots,(n+3) / 2,2)$ имеет длину $(n+1) / 2$, и лемма 3 в этом случае справедлива.

3. Пусть $g_{2}((n+3) / 2)=1$ (см. рис. 7).

Если $g_{2}(1) \in\{(n+7) / 2,(n+9) / 2, \ldots, n-1,0\}$, то контур $\left(1, g_{2}(1), g_{2}(1)+1, \ldots, 0,1\right)$ имеет длину, не большую $(n-3) / 2$, что противоречит условию теоремы 1 .

Если $g_{2}(1) \in\{5,6, \ldots,(n+1) / 2,(n+3) / 2$,$\} , то контур$

$$
\left(1, g_{2}(1), g_{2}(1)+1, \ldots,(n+1) / 2,(n+3) / 2,1\right)
$$

имеет длину, не большую $(n-3) / 2$, что противоречит условию теоремы 1.

Следовательно, $g_{2}(1) \in\{(n+5) / 2,3,4\}$. 
3.1. Пусть $g_{2}(1)=4$ (см. рис. 7).

Отметим сразу, что в этом случае контур $(1,4,5, \ldots,(n+3) / 2,1)$ имеет длину $(n-1) / 2$ и второе утверждение теоремы 1 справедливо.

Если $g_{2}(4) \in\{6,7, \ldots,(n+1) / 2\}$, то контур

$$
\left(1,4, g_{2}(4), g_{2}(4)+1, \ldots,(n+1) / 2,(n+3) / 2,1\right)
$$

имеет длину, не большую $(n-3) / 2$, что противоречит условию теоремы 1.

Если $g_{2}(4) \in\{(n+13) / 2,(n+15) / 2, \ldots, n-1,0,1,2,3\}$, то контур

$$
\left(4, g_{2}(4), g_{2}(4)+1, \ldots, 2,3,4\right)
$$

имеет длину, не большую $(n-3) / 2$, что противоречит условию теоремы 1 .

Следовательно, $g_{2}(4) \in\{(n+5) / 2,(n+7) / 2,(n+9) / 2,(n+11) / 2\}$.

Если $g_{2}(4)=(n+11) / 2$, то контур $(4,(n+11) / 2,(n+13) / 2, \ldots, n-1,0,(n+3) / 2,1,4)$ имеет длину, не большую $(n-3) / 2$, что противоречит условию теоремы 1 .

Если $g_{2}(4)=(n+9) / 2$, то контур $(4,(n+9) / 2,(n+11) / 2, \ldots, n-1,0,1,2,3,4)$ имеет длину $(n+1) / 2$, и лемма 3 в этом случае справедлива.

Если $g_{2}(4)=(n+7) / 2$, то контур $(4,(n+7) / 2,(n+9) / 2, \ldots, n-1,0,(n+3) / 2,1,4)$ имеет длину $(n+1) / 2$, и лемма 3 в этом случае справедлива.

Пусть $g_{2}(4)=(n+5) / 2$. Из рис. 7 легко видеть, что наличие в графе $\Gamma$ любой дуги $((n+5) / 2, i)$ приводит к появлению в $\Gamma$ контура длины 3,4 или 5 , если $i \in\{0,2,3\}$, либо к появлению контура длины, не большей $(n-3) / 2$, если

$$
i \in\{5,6, \ldots,(n+1) / 2\} \cup\{(n+9) / 2,(n+11) / 2, \ldots, n-1\} .
$$

Получаем противоречие с условием теоремы 1 .

3.2. Пусть $g_{2}(1)=3$. Тогда контур $(1,3,4,5, \ldots,(n+1) / 2,(n+3) / 2,1)$ имеет длину $(n+1) / 2$, расстояние от вершины 2 до этого контура равно 1 , и лемма 3 в этом случае справедлива.

3.3. Пусть $g_{2}(1)=(n+5) / 2$ (см. рис. 8). Тогда $g_{2}((n+5) / 2) \in\{2,3,4\}$.

3.3.1. Пусть $g_{2}((n+5) / 2)=4$ (см. рис. 8). Тогда контур

$$
(1,(n+5) / 2,4,5, \ldots,(n+3) / 2,1)
$$

имеет длину $(n+1) / 2$, расстояние от вершин из множества $\{2,3\}$ до этого контура не превосходит 2 , и лемма 3 в этом случае справедлива.

3.3.2. Пусть $g_{2}((n+5) / 2)=3$. Тогда контур $(3,4, \ldots,(n+3) / 2,(n+5) / 2,3)$ имеет длину $(n+1) / 2$, расстояние от вершин из множества $\{1,2\}$ до этого контура не превосходит 2 , и лемма 3 в этом случае справедлива.

3.3.3. Пусть $g_{2}((n+5) / 2)=2$ (см. рис. 9). Повторяя рассуждения начала пункта 3 для вершин $1,(n+5) / 2,2$ (вместо $0,(n+3) / 2,1)$, получаем, что $g_{2}(2)=(n+7) / 2$ и $g_{2}((n+7) / 2)=3$ и т.д., вплоть до тройки вершин $((n-3) / 2, n-2,(n-1) / 2)$ (см. рис. 9).

Тогда $g_{2}(n-1)=0$, что гіротиворечит отсутствию в $\Gamma$ параллельных дуг.

С учетом всех рассмотренных случаев лемма 3 доказана.

Таким образом, теорема 1 доказана.

Следствие 1. Любой граф Г из множества $G(n, 2,(n-1) / 2)$ при нечетном $n \geqslant 13$ примитивен. 


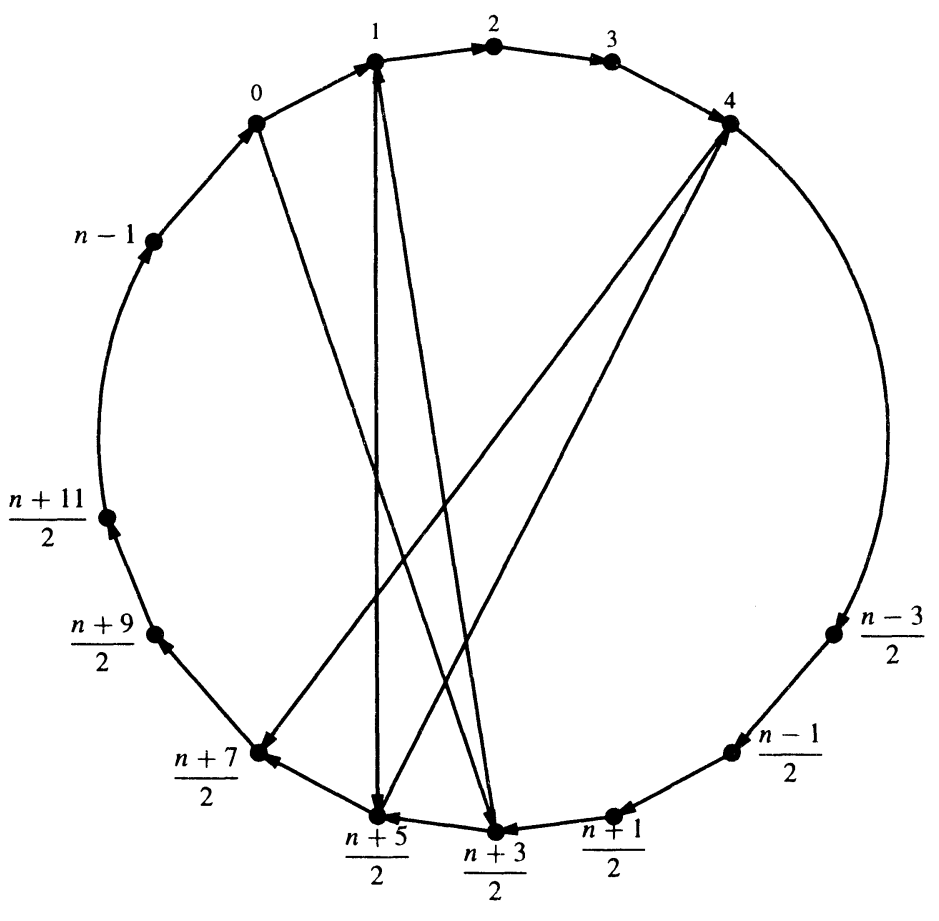

Рис. 8.

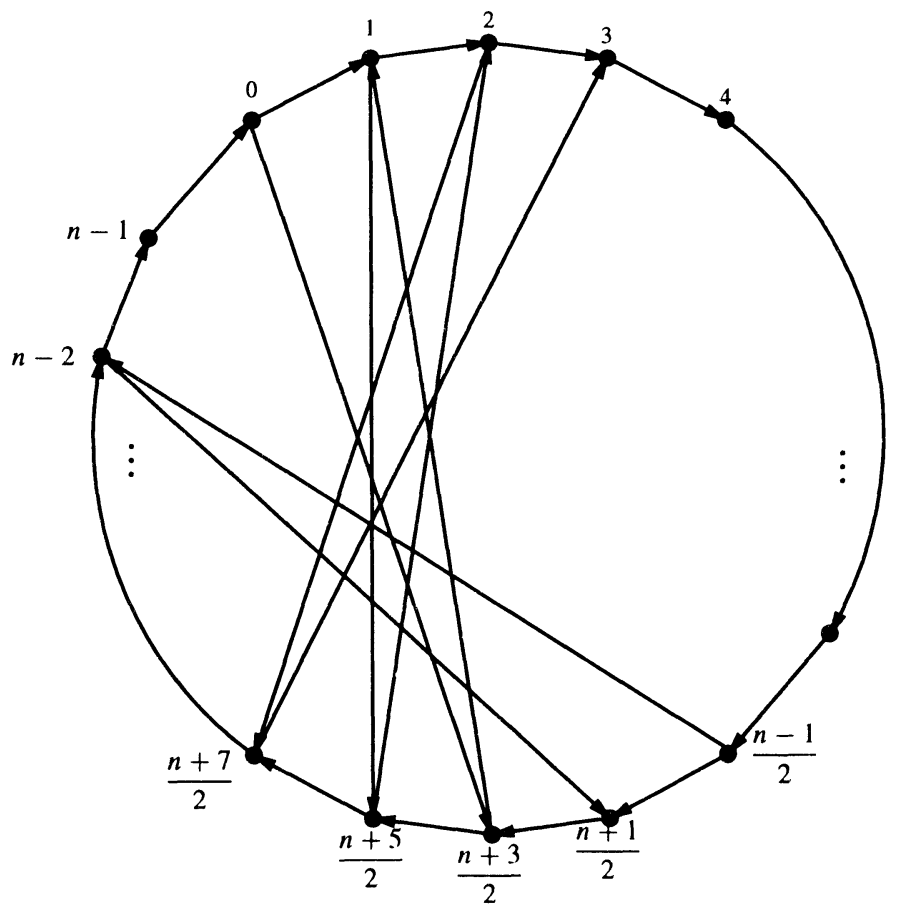

Puc. 9. 


\section{Доказательство следствия очевидно.}

Теорема 2. Если $\Gamma \in G(n, 2,(n-1) / 2), n-$ нечетное число, $n \geqslant 13$, то

$$
\gamma(\Gamma) \leqslant \frac{(n-1)^{2}}{4}+5
$$

Доказательство. Учитывая, что $\operatorname{gcd}((n-1) / 2,(n+1) / 2)=1$, положим

$$
R=\{(n-1) / 2,(n+1) / 2\} \text {. }
$$

Тогда в соответствии с (3)

$$
\gamma(\Gamma) \leqslant \rho(R)+\varphi((n-1) / 2,(n+1) / 2) .
$$

В соответствии с (2)

$$
\varphi((n-1) / 2,(n+1) / 2)=\left(\frac{n-1}{2}-1\right)\left(\frac{n+1}{2}-1\right)=\frac{(n-3)(n-1)}{4} .
$$

В следуюшей лемме оценивается $\rho(R)$.

Лемма 4. В условиях теоремы 2 справедлива оченка

$$
\rho(R) \leqslant 2+\frac{(n+1)^{2}}{2(n-2)}
$$

Доказательство. Возможны следующие два случая.

В графе Г любая пара контуров длины $(n-1) / 2$ и $(n+1) / 2$ имеет хотя бы одну общую точку (пересекается). В этом случае справедливость (9) следует непосредственно из (1) при $p=(n-1) / 2$, определения $\rho(R)$ и теоремы 1 .

В графе $\Gamma$ существует пара контуров $Q_{1}$ длины $(n+1) / 2$ и $Q_{2}$ длины $(n-1) / 2$, не имеющих общих точек. Тогда существует разложение матрицы смежности $A$ графа $\Gamma$ в сумму подстановочных матриц $\bar{g}_{1}$ и $\bar{g}_{2}$ (см. начало доказательства теоремы 1 ) такое, что одна из подстановок имеет цикловую структуру $[(n-1) / 1,(n+1) / 2]$.

Без ограничения общности можем считать, что это цикловая структура подстановки $g_{1}$ и

$$
g_{1}=\left(\begin{array}{lll}
0 & 1
\end{array} \ldots(n-1) / 2(n+1) / 2\right)((n+3) / 2 \ldots n-2 n-1) .
$$

Из соображений размерности получаем, что в этом случае все вершины контура $Q_{1}$, соответствующего циклу $(01 \ldots(n-1) / 2(n+1) / 2)$ кроме одной (без ограничения общности $i, 0 \leqslant i \leqslant(n-1) / 2)$, имеют исходящие дуги, соответствующие переходам подстановки $g_{2}$, которые входят только в вершины контура $Q_{2}$, соответствующего циклу $((n+3) / 2 \ldots n-2 n-1)$, а дуга, исходящая из $i$, может иметь только вид $(i, i+2)$ (в силу отсутствия в $\Gamma$ контуров длины, меньшей $(n+1) / 2)$ (см. рис. 10$)$.

В то же время очевидно, что все дуги, соответствующие переходам $g_{2}$, исходящие из вершин $Q_{2}$, входят только в вершины $Q_{1}$ (за исключением вершины $i+2$ ).

Из описания структуры графа $\Gamma$, оценки (1) при $p=(n-1) / 2$ и определения $\rho(R)$ следует справедливость (9) и в этом случае.

Лемма 4 доказана. 


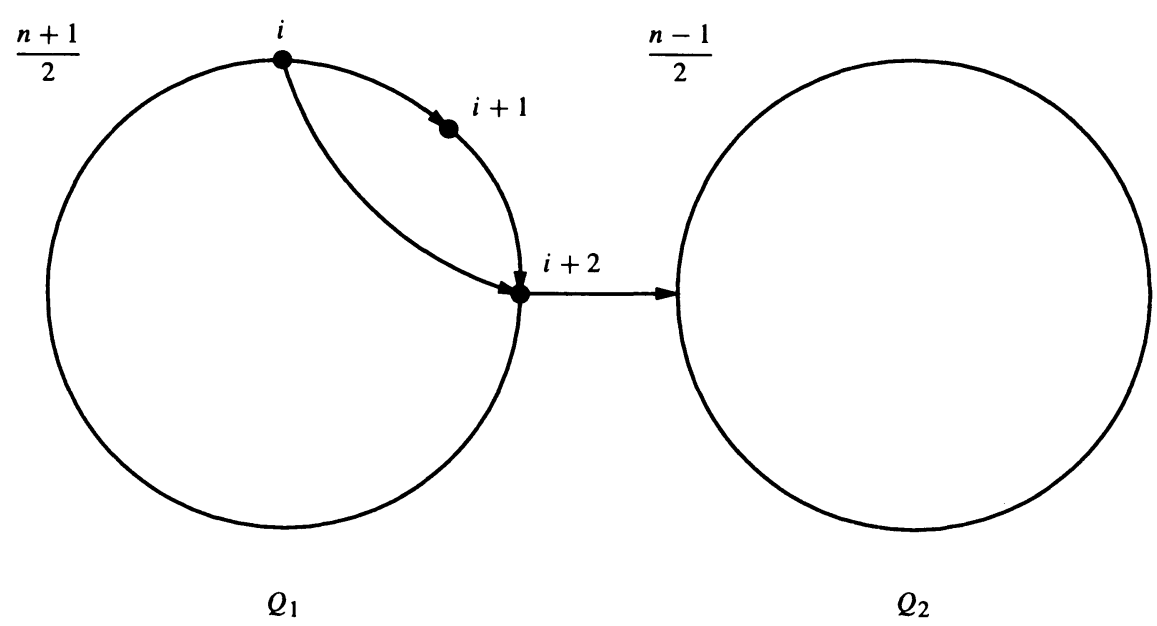

Рис. 10.

Продолжим доказательство теоремы 2. С учетом оценки из леммы 4

$$
\begin{aligned}
\gamma(\Gamma) & \leqslant \frac{(n-3)(n-1)}{4}+\frac{(n+1)^{2}}{2(n-2)}+2 \\
& =\frac{(n-3)(n-1)}{4}+\frac{n^{2}-2 n+4 n-8+9}{2(n-2)}+2 \\
& =\frac{(n-3)(n-1)}{4}+\frac{(n-2)(n+4)}{2(n-2)}+\frac{9}{2(n-2)}+2 .
\end{aligned}
$$

Тогда, учитывая условие $n \geqslant 13$, получаем, что

$$
\begin{aligned}
\gamma(\Gamma) & \leqslant \frac{(n-3)(n-1)}{4}+\frac{n+4}{2}+\frac{1}{2}+2 \\
& =\frac{(n-3)(n-1)}{4}+\frac{n-1}{2}+3+2=\frac{(n-1)^{2}}{4}+5 .
\end{aligned}
$$

Теорема 2 доказана.

Достижимость оценки характеризует следующий результат (см. рис. 11).

Теорема 3. Для экспонента графа $\Gamma^{*} \in G(n, 2,(n-1) / 2)$ справедливо равенство

$$
\gamma\left(\Gamma^{*}\right)=\frac{(n-1)^{2}}{4}+1
$$

Доказательство теоремы приведено в [4]. 


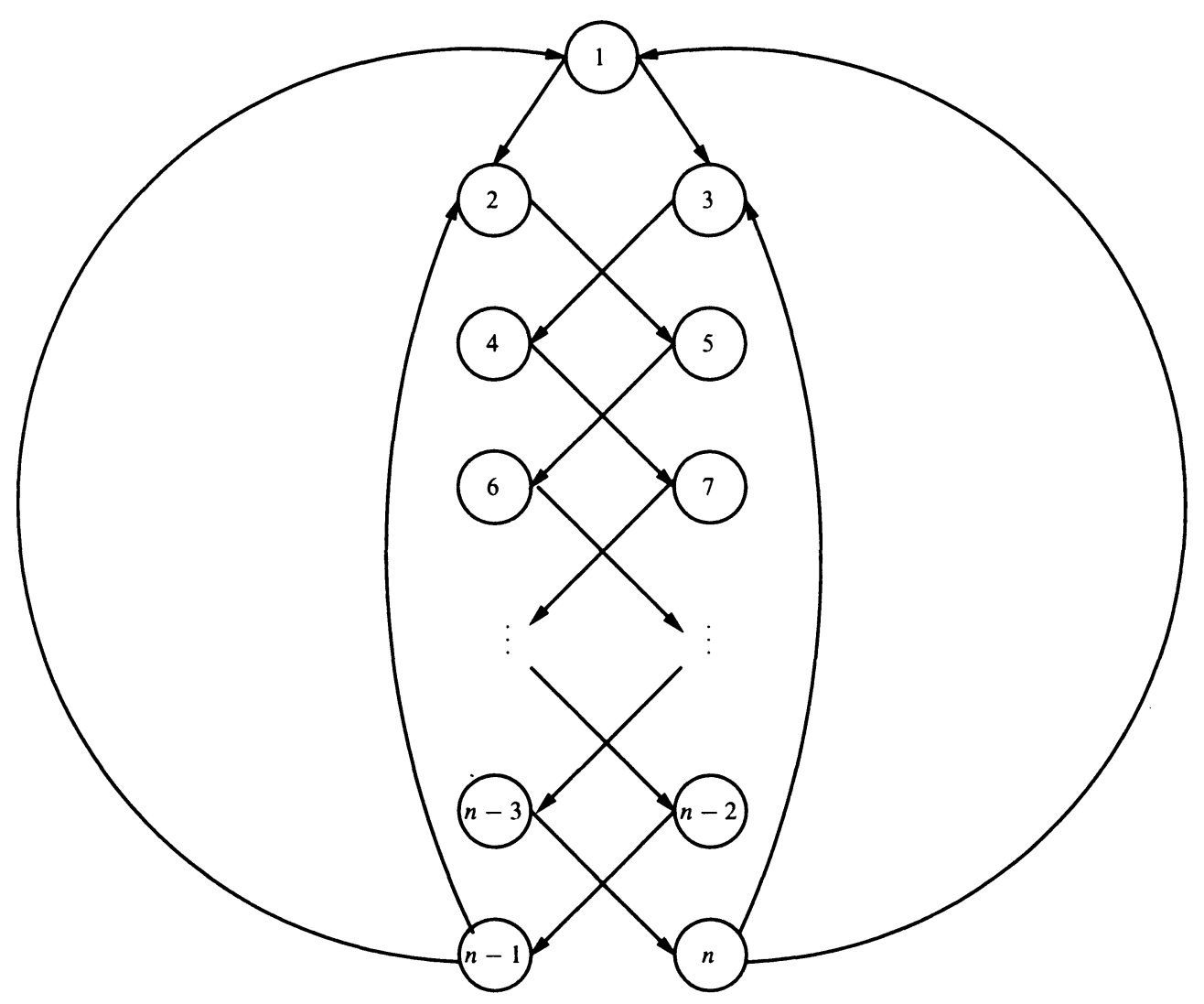

Рис. 11.

\section{Список литературы}

1. Гантмахер Ф. Р., Теория матрич. Наука, Москва, 1988.

2. Князев А. В., Верхние оценки экспонентов псевдосимметрических графов. Дискретная математика (1990) 2, №4, 63-71.

3. Князев А. В., Дихотомические графы с максимальным обхватом. Дискретная математика (1990) 2, №3, 56-64 .

4. Князев А. В., О диаметрах дихотомических графов. Матем. заметки (1992) 50, №1, 46-55.

5. Behzad M., Minimal 2-regular digraphs with given girth. J. Math. Soc. Japan (1973), №5, 1-6.

6. Shao Jia-Yu, On the exponent of a primitive digraph. Linear Algebra and its Appl. (1985) 64, 21-31.

7. Sylvester J. J., Math questions and their solutions. Educational Times (1884) 41, 21.

8. Vitek Y., Bound for a linear diophantine problem of Frobenius. J. London Math. Soc. (1975), №10, 79-85. 